\title{
Acquired Intergenic ANK3-RET Fusion as a Pralsetinib-Responsive Mechanism of Resistance to Osimertinib in EGFR-Mutated NSCLC
}

\author{
Edyta Maria Urbanska ${ }^{1 *}$, Jens Benn Sørensen ${ }^{1}$, Linea Cecilie Melchior ${ }^{2}$, Junia Cardoso Costa ${ }^{3}$ and Eric Santoni-Ru- \\ giu $^{2,4 *}$
}

1 Department of Oncology, Rigshospitalet, University of Copenhagen, 2100 Copenhagen, Denmark; Edyta.Maria.Urbanska@regionh.dk; jens.benn.soerensen@rh.regionh.dk

2 Department of Pathology, Rigshospitalet, University of Copenhagen, 2100 Copenhagen, Denmark; linea.cecilie.melchior@regionh.dk; eric.santoni-rugiu.02@regionh.dk

3 Department of Radiology, Rigshospitalet, University of Copenhagen, 2100 Copenhagen, Denmark junia.cardoso.costa@regionh.dk

4 Biotech Research \& Innovation Center (BRIC), University of Copenhagen; eric.santoni-rugiu@bric.ku.dk

* Correspondence: Edyta.Maria.Urbanska@regionh.dk; eric.santoni-rugiu.02@regionh.dk

\begin{abstract}
:
The unavoidable progression of EGFR-mutated NSCLC on EGFR-TKIs forces us to discover solutions for further therapies. Herein, we discuss the necessity of accurate genomic mapping of progressive disease illustrated by a patient case. Tumor rebiopsies at progression are strongly needed to characterize acquired resistance to EGFR-TKI. The necessary data, however, may be reliably obtained only by deep targeted next generation sequencing (NGS) of both DNA and RNA. The reported case is a patient with EGFR-mutant NSCLC, who progressed during second line Osimertinib with subsequent targeted treatment determined by the detection of an acquired intergenic ANK3-RET-fusion with concomitant PTEN-mutation and MDM2-amplification. These three acquired gene alterations represent potential mechanisms of TKI-resistance, not previously reported on second line Osimertinib. Yet, while PTEN-mutations and MDM2-amplification are currently undruggable, the ANK3-RET fusion allowed further personalized treatment by combining continuation of Osimertinib with the RET-TKI Pralsetinib, which resulted in objective partial response, so far for 7 months, and significant clinical improvement. Hence, complementary DNA- and RNA-based targeted NGS may be of importance in clinical routine to better reveal the current molecular state of the disease and contribute to the identification of further targeted therapy strategies. Indeed, further cases with acquired RET-fusions, including ANK3-RET, should be investigated to fully determine the effectiveness of RETTKI-Osimertinib combinations.
\end{abstract}

Keywords: RNA-based NGS; EGFR mutated NSCLC; acquired resistance; RET-fusion; rebiopsy

\section{Introduction}

Even though the majority of patients with metastatic non-small cell lung cancer harboring EGFR (19del or L858R)-mutations respond to first line treatment with EGFR-TKI, their disease inevitably progresses because of acquired resistance to the drugs. The extent of progression-free survival (PFS) depends on both the treatment used and the primary status of the disease including possible intrinsic resistance [1]. Beyond standard first line with Osimertinib providing median PFS of 18.9 months [2], there are also promising recent results with 19 months PFS by combining first generation EGRF-TKI with VEGFR- 
TKI $[3,4]$ and 16-21 months when using first generation EGFR-TKI with standard platinbased chemotherapy [5,6]. The concept of incorporating another drug in the first line is continuing in other studies investigating the efficacy of third generation EGFR-TKI with anti-VEGFR1 (NCT02971501) and anti-VEGFR2 (NCT03909334) or with chemotherapy (NEJ032C, FLAURA2). Although the subsequent genomic background for the progression may differ depending on treatment used and the innate nature of the disease, the cancer cells appear to have a relatively limited repertoire of bypass signaling pathways including abnormalities in e.g., BRAF, ALK, NTRK, RET, or ROS1 genes [7]. These genes may represent further therapeutic targets. However, detection of their abnormalities may require both DNA- and RNA-based NGS.

\section{Results}

We present an illustrative case of a Caucasian female, 62 years of age, never-smoker, who underwent intracranial surgery due to suspected pituitary adenoma. However, histopathological assessment and DNA-based NGS of the removed pituitary lesion revealed instead a metastasis from a pulmonary adenocarcinoma (immunohistochemically CK7+/TTF1+) with the EGFR p.E746_A750delELREA microdeletion in exon 19 (ex19del) and negative PD-L1 expression. In addition, a tumor in the left pulmonary lobe was detected together with several metastatic infiltrates in both lungs, mediastinal lymph nodes, and in the vertebral corpus of L2, corresponding to T3N2M1c. MRI of the cerebrum showed the metastasis in the pituitary gland and a smaller one posteriorly in the frontal lobe. The patient received stereotactic radiosurgery (SRK) against both intracranial processes followed by Erlotinib and achieved intracranial partial response (PR).

Intrathoracic progression was observed 15 months later and NGS analysis of DNA isolated from the rebiopsy of progressive lesions in the left lung's upper lobe and N2 lymph nodes (station 7) revealed the original EGFR ex19del as well as an acquired EGFR mutation p.T790M. Circulating free DNA (cfDNA) from plasma was without EGFR mutations. The patient started second line Osimertinib $80 \mathrm{mg}$ QD. Plasma cfDNA sampled at 4, 7 and 13 months after starting this treatment showed no presence of circulating EGFR-mutated clones, however new progression with liver metastases was observed after 15 months. DNA-NGS of tumor rebiopsy from one of these new hepatic lesions showed persistent EGFR ex19del and p.T790M mutation, which also reappeared in concomitantly sampled plasma cfDNA. In addition, MDM2-amplification (19 gene copies), and the missense PTEN p.C124S substitution (pathogenic/oncogenic mutation in the phosphatase catalytic domain of PTEN according to COSMIC [8]andOncoKB databases [9]) were found in the hepatic metastasis. Based on these two presumed resistance mutations (MDM2 sequesters and inhibits the p53 tumor suppressor protein necessary for EGFR-TKI-mediated apoptosis, while the PTEN mutation activates the PI3K/Akt pathway downstream EGFR), no further targeted treatment options were possible. The patient started chemotherapy with Carboplatin/Pemetrexed, while continuing Osimertinib. Three cycles of this treatment induced stable disease but was poorly tolerated due to bone marrow toxicity and the patient did not tolerate more chemotherapy. The patient was supposed to be without further therapeutic options. However, additional NGS analysis of RNA isolated from the hepatic metastasis showed ANK3-RET fusion on chromosome 10q with breakpoints breakpoint chr10: 61994446 on negative strand and chr10: 43612032 on positive strand (Figure 1). The employed NGS method, which sequences cDNAs from mRNA products, predicted the fusion to be in frame and could detect it in $71.5 \%$ of reads. According to the annotations in COSMIC database [8], both breakpoints are outside the coding sequence of the two genes on chromosome 10q (RET: chr10:43077027-43130351, negative strand; ANK3: chr10: 60026298-60389730, positive strand) and other genes are present on chromosome 10q between ANK3 and RET; suggesting that the ANK3-RET fusion was intergenic and possibly generated by an inversion or a loop on chromosom 10q. Retrospective NGS analysis of RNA isolated from the pre-Osimertinib biopsies (diagnostic pituitary biopsy at baseline 
and tumor re-biopsy obtained at the first intrathoracic progression) did not show the ANK3-RET fusion, confirming that the latter had been acquired during treatment with Osimertinib.

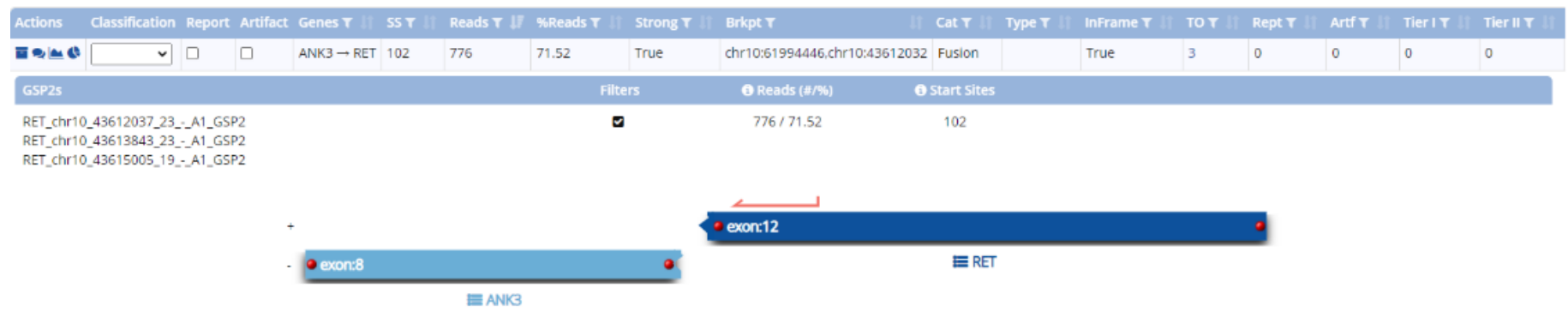

Figure 1. Visualization of the intergenic ANK3-RET fusion on chromosome 10q identified by RNA-NGS and the Archer analysis software (breakpoint chr10: 61994446 for RET on negative strand, chr10: 43612032 for ANK3 on positive strand).

This finding may further explain the progression on Osimertinib, as other types of RET-fusions have been reported as mechanisms of acquired resistance to first line EGFR-TKIs, including Osimertinib [10-12]. Thus, RET-TKI treatment with Pralsetinib $400 \mathrm{mg}$ QD combined with continuation of Osimertinib $80 \mathrm{mg}$ QD was initiated.

Table 1. Treatment lines with the corresponding molecular state of the disease in each rebiopsy, and detection method used to reveal the genetic abnormalities in the tumor tissue.

\begin{tabular}{|c|c|c|c|c|}
\hline TREATMENT LINES & $\begin{array}{c}\text { START } \\
\text { DATE }\end{array}$ & MOLECULAR FINDINGS & $\begin{array}{c}\text { METHOD } \\
\text { USED* }\end{array}$ \\
\hline 1 & Erlotinib & 2018 & EGFR ex19del & DNA-NGS \\
\hline 2 & Osimertinib & 2019 & $\begin{array}{c}\text { EGFR ex19del; } \\
\text { EGFR T790M }\end{array}$ & DNA-NGS \\
\hline 4 & $\begin{array}{c}\text { Osimertinib } \\
\text { chemotherapy } \\
4\end{array}$ & 2020 & $\begin{array}{c}\text { EGFR ex19del; EGFR } \\
\text { T790M; PTEN C124S } \\
\text { MDM2 amplification }\end{array}$ & DNA- NGS \\
\hline Pralsetinib & 2021 & ANK3-RET fusion & RNA-NGS \\
\hline
\end{tabular}

\footnotetext{
*Theindicated methods were used up-front. However, the diagnostic biopsy and the following rebiopsies were retrospectively reassessed using RNA-NGS as well.
} 


\section{Tumour journey}

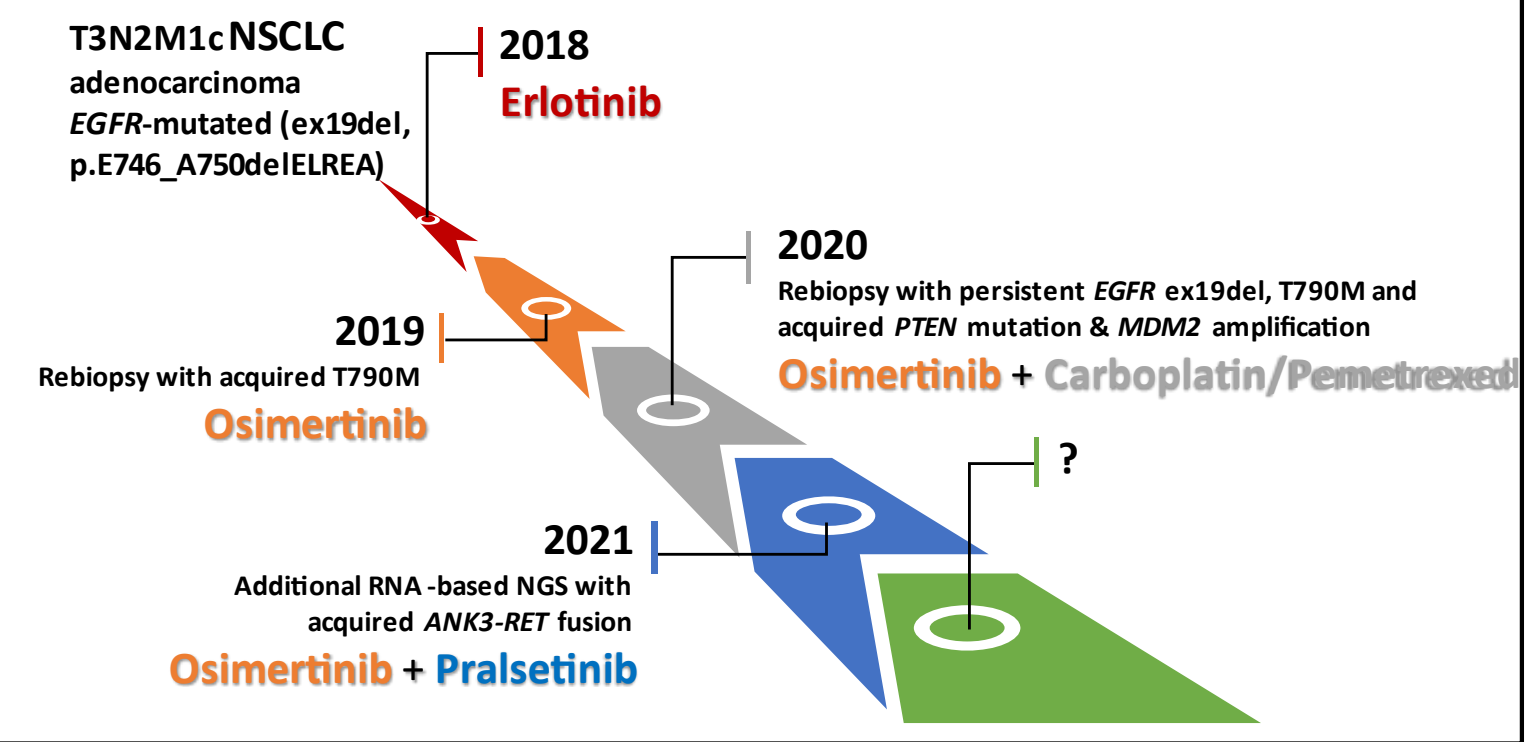

Figure 2. Tumor journey illustrating multiclonality of the disease during progression and adopted treatments. As the patient is still responding to Osimertinib-Pralsetinib combination, the future molecular state of the disease remains to be explored (symbol "?").

After two weeks of combined treatment with EGFR-TKI and RET-TKI the patient was hospitalized due to dyspnea grade 3 with PS 2 and desaturation reduced to $86 \%$, but without fever. A chest CT scan revealed new diffusely widespread ground-glass opacities in the right lung and less prevalent in the left upper lobe (Fig. 3a). The clinical findings and radiological changes were assessed to represent interstitial pneumonitis.
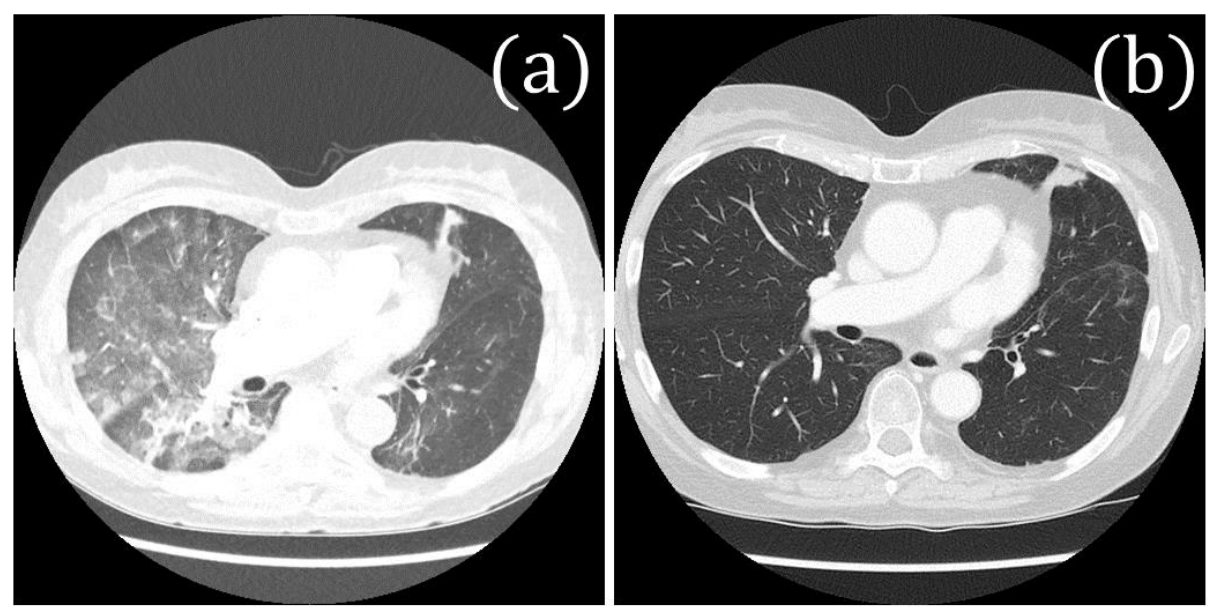

Figure 3. Thoracic CT-scan illustrating the drug induced pneumonitis two weeks after EGFR-TKI + RET-TKI combination. a) Drug induced pneumonitis: areas with ground-glass opacities and air 
space consolidation in both lungs (most pronounced in the right side), (b) resolution of pneumonitis: the areas with parenchymal opacity disappeared in both lungs.

The treatment with Osimertinib and Pralsetinib was paused, and the patient started high dose of steroids $(1 \mathrm{mg} / \mathrm{kg})$ with significant improvement and was discharged a couple of days later.

The control CT scan showed total regression of the pneumonitis (Fig. 3b), so that the patient resumed the combined TKI-treatment in reduced dose (Osimertinib $40 \mathrm{mg}$ QD and Pralsetinib $200 \mathrm{mg}$ QD). The patient continued this treatment without additional adverse events and with further clinical improvement. At the first assessment performed 1 month after the treatment was resumed, a mixed response was observed since the primary tumor in the left lung's upper lobe had regressed, one liver metastasis disappeared, while a growth of other liver metastases occurred (Fig. 4a-c vs. $4 \mathrm{~d}-\mathrm{f}$ ). At the second assessment 4 weeks later, further reduction of the primary tumor was observed together with regression of liver metastases (Fig. 4g-i). Stable disease, both in chest and abdomen was maintained at the third and fourth assessment performed after 5 months (Fig.4j-1) and 7 months of treatment (data not shown), respectively. In concordance with the radiological response, sequential samplings of plasma cfDNA did not show reappareance of circulating EGFR-mutated clones during the combination treatment. 


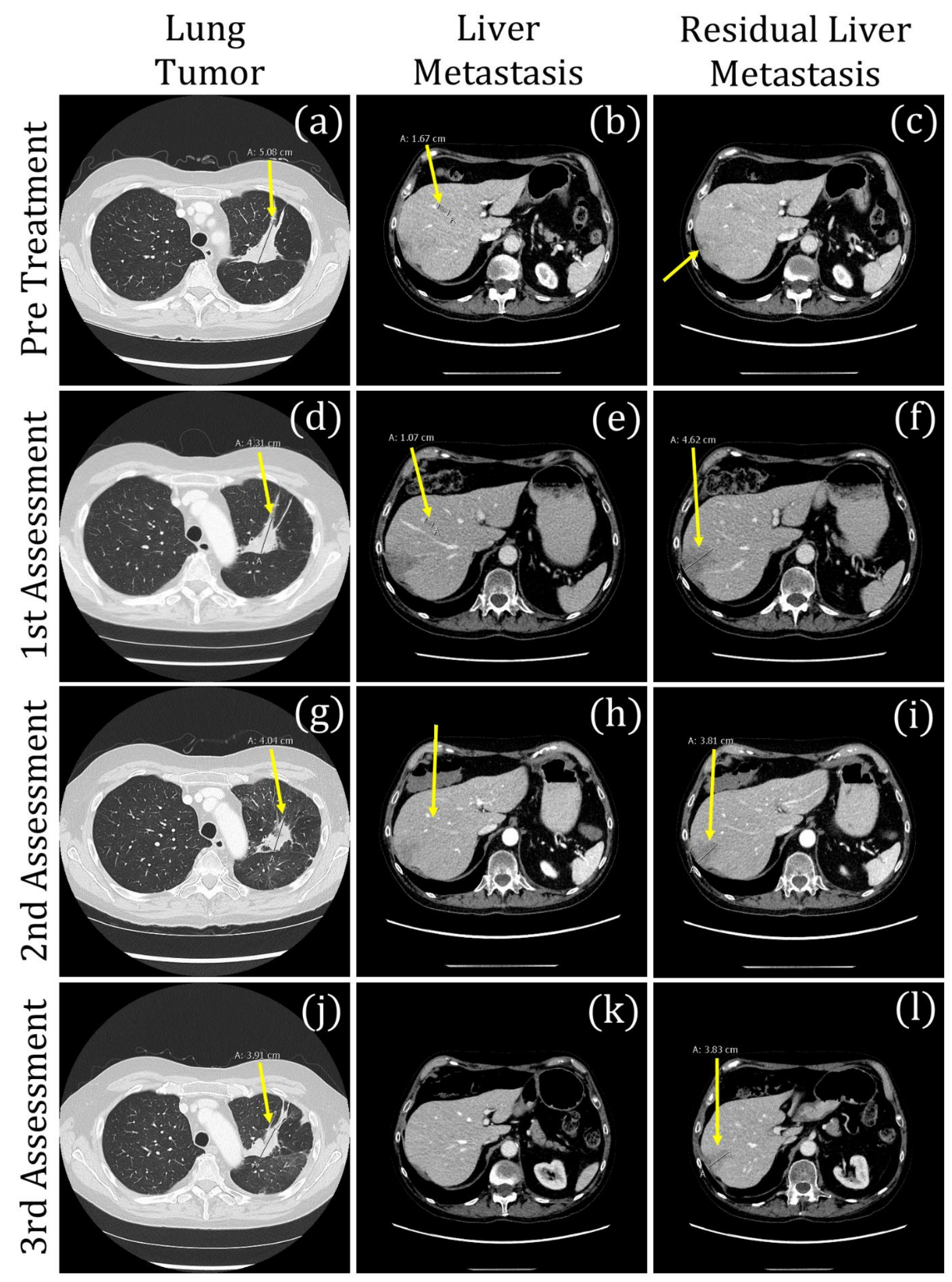

Figure 4. Response to combined treatment with Osimertinib and Pralsetinib. Pre-treatment: (a) lung tumor in left upper lobe $(5.1 \mathrm{~cm})$, (b) liver metastasis $(1.7 \mathrm{~cm})$, (c) residual liver metastasis (non-measurable). First 
assessment: (d) lung tumor in left upper lobe $(4.3 \mathrm{~cm}),(\mathrm{e})$ liver metastasis $(1.1 \mathrm{~cm}),(\mathrm{f})$ residual liver metastasis $(4.6 \mathrm{~cm})$. Second assessment: (g) lung tumor in left upper lobe $(4.0 \mathrm{~cm}),(\mathrm{h})$ liver metastasis (nonmeasurable), (i) residual liver metastasis $(3.8 \mathrm{~cm})$. Third assessment: (j) lung tumor in left upper lobe (3.9 $\mathrm{cm}),(\mathrm{k})$ liver metastasis (non-visible), (l) residual liver metastasis $(3.8 \mathrm{~cm})$.

\section{Discussion}

In the current era of rapid development of new techniques and new treatment targets, new molecular testing strategies are needed. In a prospective study of clinical genomic testing among 2,522 lung adenocarcinomas further RNA-testing revealed additional 14\% druggable alterations that had not been detected by DNA-based testing, but also enriched DNA driver-negative samples [13]. The study was to our knowledge the first extensive evidence on the importance of complementary RNA-based testing for finding potential new treatment options.

RET-fusion as acquired resistance mechanism has previously been sporadically reported in patients progressing on Osimertinib [12]. In a group of forty-one patients with rebiopsies performed at progression on first line Osimertinib, two patients were identified with acquired CCDC6-RET fusion (4.8\%). They were successfully treated with a RET-TKI (Pralsetinib), while continuing Osimertinib [12]. The biological role of acquired RET-fusion as an oncogenic driver mediating resistance was also evaluated. The authors observed that NSCLC cell lines expressing CCDC6-RET fusion became resistant to EGFRTKI but could be resensitized by using selective RET inhibitors.

The combination of Osimertinib with another RET-TKI, Selpercatinib, for a group of EGFR-mutated NSCLC patients with acquired RET-fusions with different partners such as NCOA4, CCDC6 4, KIF5B, and RUFY2, turned out to be effective with ORR of $50 \%$ and median duration of treatment being 7,4 months [14]. In our case, after progression on $2^{\text {nd }}$ line Osimertinib, we have detected the acquired fusion of RET with another partner, ANK3. Furthermore, after combining Pralsetinib to Osimertinib, we have observed an objective partial response that so far has been maintained for 7 months of combined treatment, in line with the data by Rotow et al. [14]. Together, these findings strongly indicate that RET-fusions may mediate resistance to EGFR-TKI therapy and that the combination of Osimertinib with a RET-TKI may counteract RET-mediated resistance to Osimertinib.

The rebiopsy of our patient was examined histologically without finding any changes in tumor phenotype (i.e., no transformation to SCLC or squamous carcinoma, or epithelial-mesenchymal transition) that could represent mechanisms of resistance to EGFR-TKIs [1]. Subsequently, DNA-NGS analysis of SNVs, indels and CNVs across 161 unique cancer-associated genes was performed to find possible by-pass resistance mechanisms that could explain the progression on Osimertinib. Although, we did identify putative mechanisms of TKI-resistance, such as PTEN-mutation and MDM2-amplification, no additional targeted treatment could be offered to the patient based on these results. The only treatment option in such situations was chemotherapy. Immunotherapy could also be considered, but it was not possible in our patient receiving steroid substitution due to hypophysis insufficiency. Furthermore, there are no convincing data that support the treatment with PD-(L)1 inhibitor in patients with activating EGFR mutations [15].

However, further testing with RNA-NGS resulted in identification of acquired RETfusion. This finding significantly changed the perspective for the patient by allowing further targeted treatment combining Osimertinib with a RET-TKI. Resistance to Osimertinib varies depending on treatment lines and acquired RET-fusion is present in about $4 \%$ of re-biopsied cases, representing one of the four reported off-target resistance mechanisms to first line Osimertinib, beside MET-amplification, BRAF-fusion, and KRAS-mutation [1, 16,17]. Our patient showed acquired RET-fusion after progression on second line Osimertinib. This distinct ANK3-RET fusion has not been reported before as mechanism of acquired Osimertinib resistance. Hitherto, forty-eight different RET-fusions partners have been reported, occurring both as de novo and as acquired resistance during treatment with 
EGFR-TKI in first or further lines [18]. Notably, the ANK3-RET fusion has previously been identified in de novo setting, but not as acquired fusion [19].

Resistance mechanisms on further lines of Osimertinib are dominated by on-target events such as EGFR C797X or other rare tertiary mutations and EGFR-amplification $[16,17]$. Off-target resistance mechanisms include MET-amplification, MET-exon 14 skipping, ALK- and BRAF-fusion, as well as HER2- and KRAS-mutations [16,17]. To our knowledge, this is the first report of ANK3-RET fusion as off-target resistance mechanism in second line Osimertinib. This finding indicates the importance of current molecular strategy in clinical practice, where further RNA-based testing is needed. However, the acquired ANK3-RET fusion in our case was accompanied by two other previously reported resistance mechanisms: PTEN mutation and MDM2 amplification. The PTEN tumor suppressor acts as negative regulator of the PI3K/AKT/mTOR pathway. Up-front loss-of-function mutations of PTEN are relatively common in NSCLC, especially in squamous cell carcinoma $(10,2 \%)$ and are reportedly linked with worse prognosis [,20,21]. Moreover, co-existing de novo PTEN-mutations at baseline in patients with metastatic, EGFR-mutated, pulmonary adenocarcinoma are a predictor of short PFS during first line therapy with EGFR-TKIs [22] and may contribute to resistance to Erlotinib [23]. Variants in the PTEN coding sequence are uncommon in most types of cancer $(<10 \%)$, nevertheless they can be detected in about $8 \%$ of NSCLC cases [24]. The acquired PTEN substitution identified in our patient has not been described before under Osimertinib treatment and may function as a parallel resistance mechanism alongside the RET-fusion.

The third co-existing potential mechanism of resistance that we found in our case was high-level amplification of the MDM2 oncogene (19 copies). MDM2 interacts with many proteins, most importantly, it functions as an E3 ubiquitin-ligase that targets the p53 tumor suppressor for proteolysis and can antagonize its transcriptional activation [25]. MDM2-amplification is associated with EGFR-TKI resistance. In a retrospective cohort of seventy-five patients with EGFR-mutated NSCLC treated with first, second or third generation EGFR-TKIs, pre-existing MDM2-amplification was associated with worse outcome [26]. Neither PTEN-mutation nor MDM2-amplification were found in the diagnostic biopsy or the first rebiopsy from our patient (Figure 2), thus we considered them as acquired TKI-resistance mechanisms together with the ANK3-RET fusion. These three acquired alterations reflect the clonal heterogeneity of advanced EGFR-mutated NSCLC at progression on TKIs. Despite experimental pre-clinical attempts to target alterations in the MDM2/p53 or the PTEN/PI3K/AKT/mTOR axis $[27,28]$, these pathways, in contrast to RET-fusions, remain undruggable in NSCLC. Yet, our patient's disseminated NSCLC has been exhibiting prolonged partial response to the Pralsetinib-Osimertinib combination, even in presence of MDM2-amplification and PTEN-mutation, further indicating that the ANK3-RET fusion is an important and targetable mechanism of resistance to Osimertinib.

\section{Materials and Methods}

Ten ng of genomic DNA were purified from each formalin-fixed paraffin-embedded (FFPE) biopsy using the Maxwell RSC DNA FFPE kit (Promega, Madison, USA) and were quantified by the Qubit ${ }^{\circledR}$ dsDNA HS assay on a Qubit ${ }^{\circledR} 2.0$ Fluorometer (ThermoFisher Scientific, Waltham, USA). DNA-NGS analysis of SNVs, indels and CNVs across 161 unique cancer-associated genes was conducted on 10 ng DNA by Oncomine Comprehensive Assay v3 on the Ion Torrent ${ }^{\mathrm{TM}}$ S5+system (ThermoFisher Scientific, Waltham, USA) according to the manufacturer's instructions. Additionally, liquid biopsies (cfDNA from plasma) were analyzed for relevant DNA-mutations by the Oncomine Lung cfDNA NGS-assay, as indicated by the manufacturer (ThermoFisher Scientific, Waltham, USA). For analysis of gene fusions, total RNA was extracted from the abovedescribed biopsies using Maxwell RSC RNA FFPE kit (Promega, Madison, USA). RNA- 
NGS was performed using the Archer FusionPlex ${ }^{\circledR}$ Lung kit and analysis software according to the manufacturer's instructions (ArcherDX, Inc., Boulder, USA).

\section{Conclusions}

Our and previous reports, as well as currently available different methodologies for detecting gene fusions, support the use of targeted RNA-NGS as a new standard molecular strategy that can complement DNA-NGS in the molecular assessment of both diagnostic biopsies and rebiopsies from EGFR-mutated NSCLC patients receiving Osimertinib [29]. Indeed, our case provides a practical example of how testing of rebiopsies from patients progressing on Osimertinib with both DNA- and RNA-NGS allows the identification of a broader spectrum of co-existing resistance mechanisms, and thereby a better choice of further therapeutic strategies. Furthermore, our report demonstrates that the ANK3-RET fusion is a druggable mechanism of acquired resistance to Osimertinib and can be tackled by targeted combination treatment. Thus, our data may be used for updating the clinically relevant information in the recently published catalog of intergenic RETrearrangements [18]. Finally, further cases with acquired RET-fusions, including ANK3RET, should be investigated to fully determine the effectiveness of RET-TKI-Osimertinib combinations.

Author Contributions: Conceptualization E.M.U., J.B.S., E.S.-R.; formal analysis, E.M.U., L.C.M., E.S.-R.; investigation, E.M.U., L.C.M., J.C.C., E.S.-R.; resources, E.M.U., L.C.M.; writing-original draft preparation, E.M.U.; writing - review and editing, E.M.U., J.B.S., E.S.-R.; visualization, E.M.U., L.C.M., J.C.C., E.S.-R.. All authors have read and agreed to the published version of the manuscript.

Funding: This research received no external funding.

Institutional Review Board Statement: The study was conducted according to the guidelines of the Declaration of Helsinki and approved by the National Tumor Board Phase 1 and Lung Team Tumor Board (20200122) at Department of Oncology, Rigshospitalet, University of Copenhagen.

Informed Consent Statement: Written informed consent has been obtained from the patient to publish this paper.

Data Availability Statement: All data generated during and/or analyzed during the current study, are available from the corresponding authors on reasonable request within the legal use of confidential data. The data are not publicly available due to Danish privacy law restrictions.

Conflicts of Interest: E.M.U. participated in Advisory Board (Pfizer, Amgen) received speaker honoraria (Takeda, AstraZeneca, and Roche). J.B.S. participated in Advisory Boards (Roche; AstraZeneca, Takeda, Pfizer) and received speaker honoraria (Takeda, AstraZeneca, Roche), E.S.-R. participated in Advisory Boards (Amgen, AstraZeneca, Bayer, Pfizer, Roche, Takeda) and received speaker honoraria (Amgen, Pfizer, Roche, Takeda). L.C.M. has no conflict of interest. J.C.C. has no conflict of interest.

\section{References}

1. Santoni-Rugiu, E.; Melchior, L.C.; Urbanska, E.M.; Jakobsen, J.N.; Stricker, K.; Grauslund, M.; Sørensen, J.B. Intrinsic resistance to EGFR-Tyrosine Kinase Inhibitors in EGFR-Mutant Non-Small Cell Lung Cancer: Differences and Similarities with Acquired Resistance. Cancers 2019, 1, 923. doi:10.3390/cancers11070923.

2. Soria, J.C.; Ohe, Y.; Vansteenkiste, J.; Reungwetwattana, T.; Chewaskulyong, B.; Lee, K.H.; Dechaphunkul, A.; Imamura, F.; Nogami, N.; Kurata, T.; et al. FLAURA Investigators. Osimertinib in Untreated EGFR-Mutated Advanced Non-Small-Cell Lung Cancer. N. Engl. J. Med. 2018, 11, 113-125. doi:10.1056/NEJMoa1713137. 
3. Nakagawa, K.; Garon, E.B.; Seto, T.; Nishio, M.; Ponce, A.; Paz-Ares, L.; Chiu, C.H.; Park, K.; Novello, S.; Nadal, E.; et al. Ramucirumab plus erlotinib in patients with untreated, EGFR-mutated, advanced non-small-cell lung cancer (RELAY): a randomised, double-blind, placebo-controlled, phase 3 trial. Lancet Oncol. 2019, 20, 1655-1669. doi:10.1016/S1470-2045(19)30634-5.

4. Saito, H.; Fukuhara, T.; Furuya, N.; Watanabe, K.; Sugawara,, S.; Iwasawa S.; Tsunezuka, Y.; Yamaguchi, O.; Okada, M.; Yoshimori, K.; et al. Erlotinib plus bevacizumab versus erlotinib alone in patients with EGFR-positive advanced non-squamous non-small-cell lung cancer (NEJ026): interim analysis of an open-label, randomised, multicentre, phase 3 trial. Lancet Oncol. 2019, 20, 625-635.doi:10.1016/S1470-2045(19)30035-X.

5. Hosomi, Y.; Morita, S.; Sugawara, S.; Kato, T.; Fukuhara, T.; Gemma, A.; Takahashi, K.; Fujita, Y.; Harada, T.; Minato, K.; et al. Gefitinib alone versus gefitinib plus chemotherapy for non-small-cell lung cancer with mutated epidermal growth factor receptor: NEJ009 study. J. Clin. Oncol. 2020, 10, 115-123. doi:10.1200/JCO.19.01488.

6. Noronha V.; Patil V.M.; Joshi A.; Menon N.; Chougule A.; Mahajan A.; Janu A.; Purandare N.; Kumar R.; More S.; et al. Gefitinib versus gefitinib plus pemetrexed and carboplatin chemotherapy in EGFR-mutated lung cancer. J. Clin.. Oncol 2020, 38, 124-136. doi:10.1200/JCO.19.01154.

7. Doebele, RC. Acquired Resistance Is Oncogene and Drug Agnostic. Cancer Cell 2019, 14, 347-349. doi:10.1016/j.ccell.2019.09.011.

8. COSMIC (Catalogue Of Somatic Mutations In Cancer). Available online: https://cancer.sanger.ac.uk/cosmic (accessed on September $\left.3^{\text {rd }}, 2021\right)$.

9. OncoKB, MSK's Precision Oncology Knowledge Base. Available online: https://www.oncokb.org (accessed on September $3^{\text {rd }}$, 2021).

10. Offin, M.; Somwar, R.; Rekthman, N.; Benayed, R.; Chang, J.C.; Plodkowski, A.; Lui, A.J.W.; Eng, J.; Rosenblum, M.; Li, B.T.; et al. Acquired ALK and RET gene fusions as mechanism of resistance to Osimertinib in EGFR-mutant lung cancer. JCO Precis. Oncol. 2018, 2:PO.18.00126. doi:10.1200/PO.18.00126.

11. Klempner, S.J.; Bazhenova, L.A.; Braiteh, F.S.; Nikolinakos, P.G.; Gowen, K.; Cervantes, C.M.; Chmielecki, J.; Greenbowe, J.R.; Ross, J.S. Stephens, P.J. et al. Emergence of RET rearrangement co-existing with activated EGFR-mutation in EGFR-mutated NSCLC-patients who has progressed on first or second generation EGFR-TKI. Lung Cancer 2015, 89, 357-359. doi:10.1016/j.lungcan.201 5.06.021.

12. Piotrowska, Z.; Hideko, I.; Lennerz, A.K.; Gainor, J.F.; Lennes, I.T.; Zhu, V.W.; Marcoux, N.; Banwait, M.K.; Digumarthy, S.R.; $\mathrm{Su}, \mathrm{W}$; et al. Landscape of acquired resistance to Osimertinib in EGFR-mutant NSCLC and clinical validation of combined EGFR and RET inhibition with Osimertinib and BLU-667 for acquired RET fusion. Cancer Discov. 2018, 8, 1529-1539. doi:10.1158/2159-8290.CD-18-1022.

13. Benayed, R.; Offin, M.; Mullaney, K.; Sukhadia, P.; Rios, K.; Desmeules, P.; Ptashkin, R.; Won, H.; Chang, J.; Halpenny, D.; et al. High Yield of RNA Sequencing for Targetable Kinase Fusions in Lung Adenocarcinomas with No Mitogenic Driver Alteration Detected by DNA Sequencing and Low Tumor Mutation Burden. Clin. Cancer Res. 2019, 1, 4712-4722. doi:10.1158/10780432.CCR-19-0225.

14. Rotow, J.; Patel, J.; Hanley, M.; Yu, H., Goldman, J.; Nechustan, H.; Scheffler, M; Awad, M.; Clifford, S.; Santucci, A.; et al. Combination Osimertinib plus Selpercatinib for EGFR-mutant Non-Small Cell Lung Cancer with Acquired RET Fusions. In J. Thorac. Oncol. Vol. 16, Issue 3, Supplement, S230, March 01, 2021, Proceedings of World Conference on Lung Cancer 2020, Singapore January 28-30, 2021, Worldwide Virtual Event, Abstract number FP14.07, doi.org/10.1016/j.jtho.2021.01.150.

15. Melosky, B.; Juergens, R.; Hirsh, V.; McLeod, D.; Leighl, N.; Tsao, M.S., Card, P.B.; Chu, Q. Amplifying Outcomes: Checkpoint Inhibitor Combinations in First-Line Non-Small Cell Lung Cancer. Oncologist 2020, 25, 64-77. doi:10.1634/theoncologist.20190027.

16. Schoenfeld, A.J.; Chan, J.M.; Kubota, D.; Sato, H.; Rizvi, H.; Daneshbod, Y.; Chang, J.C.; Paik, P.K.; Offin, M.; Arcila, M.E. et al. Tumor Analyses Reveal Squamous Transformation and Off-Target Alterations as Early Resistance Mechanisms to First line Osimertinib in EGFR-Mutant Lung Cancer. Clin. Cancer Res. 2020, 1, 2654-2663. doi:10.1158/1078-0432.CCR-19-3563.

17. Leonetti, A.; Sharma, S.; Minari, R.; Perego, P.; Giovannetti, E.; Tiseo, M. Resistance mechanisms to Osimertinib in EGFR-mutated non-small cell lung cancer. Br. J. Cancer 2021, 121, 725-737. doi:10.1038/s41416-019-0573-8.

18. Ou, S.I.; Zhu, V.W. Catalog of 5 'fusion partners in RET+ NSCLC Circa 2020. JTO Clin. Res. Rep. 2020, 1, 1-7. doi:10.1016/j.jtocrr.2020.100037.

19. Lu, C.; Dong, X.R.; Zhao, J.; Zhang, X.C.; Chen, H.J.; Zhou, Q.; Tu, H.Y.; Ai, X.H.; Chen, X.F.; An, G.L.; et al. Association of genetic and immuno-characteristics with clinical outcomes in patients with RET-rearranged non-small cell lung cancer: a retrospective multicenter study. J. Hematol. Oncol 2020, 15, 37. doi:10.1186/s13045-020-00866-6.

20. Jin, G.; Kim, M.J.; Jeon, H.-S.; Choi, J.E.; Kim, D.S.; Lee, E.B.; Cha, S.I. Yoon, G.S.; Kim, C.H.; Jung, T.H.; et al. PTEN-mutations and relationship to EGFR, ERBB2, KRAS, and TP53 mutations in non-small cell lung cancers. Lung Cancer 2010, 69, 279-83. doi:10. 1016/j.lungcan.2009.11.012.

21. Ferrara, M.G.; Martini, M.; D'Argento, E.; Forcella, C.; Vita, E.; Di Noia, V.; Sperduti, I.; Bilotta, M.; Ribelli, M.; Damiano, P.; et al. PTEN Loss as a Predictor of Tumor Heterogeneity and Poor Prognosis in Patients With EGFR-mutant Advanced Non-smallcell Lung Cancer Receiving Tyrosine Kinase Inhibitors. Clin. Lung Cancer 2021, 22, 351-360. doi:10.1016/j.cllc.2020.12.008.

22. Blons, H.; Oudart, J.B.; Merlio, J.P.; Debieuvre, D.; de Fraipont, F.; Audigier-Valette, C.; Escande, F.; Hominal, S.; Bringuier, P.P.; Fraboulet-Moreau, S.; et al. PTEN, ATM, IDH1 mutations and MAPK pathway activation as modulators of PFS and OS in patients treated by first line EGFR TKI, an ancillary study of the French Cooperative Thoracic Intergroup (IFCT) Biomarkers France project. Lung Cancer 2021, 151, 69-75. doi:10.1016/j.lungcan.2020.11.008. 
23. Sos, M.L.; Koker, M.; Weir, B.A.; Heynck, S.; Rabinovsky, R.; Zander, T.; Seeger, J.M. Weiss, J. Fischer, F.; Frommolt, P. et al. PTEN loss contributes to erlotinib resistance in EGFR-mutant lung cancer by activation of Akt and EGFR. Cancer Res. 2009, 69, 3256-3261. doi:10.1158/0008-5472.CAN-08-4055.

24. Álvarez-Garcia, V.; Tawil, Y.; Wise, H.M.; Leslie, N.R. Mechanisms of PTEN loss in cancer: It's all about diversity. Semin. Cancer Biol. 2019, 59, 66-79. doi: 10.1016/j.semcancer.2019.02.001.

25. Hou, H.; Sun, D.; Zhang, X. The role of MDM2 amplification and overexpression in therapeutic resistance of malignant tumors. Cancer Cell Int. 2019, 19,216. doi:10.1186/s12935-019-0937-4.

26. Kim, Y.; Lee, B.; Shim, J.H.; Lee, S.H.; Park, W.Y.; Choi, Y.L.; Sun, J.M.; Ahn, J.S.; Ahn, M.J.; Park, K. Concurrent Genetic Alterations Predict the Progression to Target Therapy in EGFR-Mutated Advanced NSCLC. J. Thorac. Oncol. 2019, 14, 193-202. doi:10.1016/ j.jtho.2018.10.150.

27. Jiang, L.; Zawacka-Pankau, J. The p53/MDM2/MDMX-targeted therapies-a clinical synopsis. Cell Death Dis. 2020, 11, 237. doi: 10.1038/s41419-020-2445-9.

28. Gkountakos, A.; Sartori, G.; Falcone, I.; Piro, G.; Ciuffreda, L.; Carbone, C.; Tortora, G.; Scarpa, A.; Bria, E.; Milella, M.; et al. PTEN in Lung Cancer: Dealing with the Problem, Building on New Knowledge and Turning the Game Around. Cancers 2019, 9, 1141. doi: 10.3390/cancers11081141.

29. Qu, X.; Yeung, C.; Coleman, I.; Nelson, P.S.; Fang, M. Comparison of four next generation sequencing platforms for fusion detection: Oncomine by ThermoFisher, AmpliSeq by illumina, FusionPlex by ArcherDX, and QIAseq by QIAGEN. Cancer Genet. 2021, 243, 11-18. doi:10.1016/j.cancergen.2020.02.007. 